\title{
1 Quantitative analysis of signaling responses during mouse 2 primordial germ cell specification
}

3 Sophie M. Morgani ${ }^{1^{*}}$ and Anna-Katerina Hadjantonakis ${ }^{1^{*}}$

4

$5 \quad{ }^{1}$ Developmental Biology Program, Sloan Kettering Institute, Memorial Sloan Kettering Cancer

6 Center, New York, NY, 10065, USA.

$8 \quad$ *Corresponding authors:

9 Sophie M. Morgani, PhD and Anna-Katerina Hadjantonakis, PhD

10 Email: morganis@mskcc.org, hadj@mskcc.org

11

12 


\section{Abstract}

15 During early mammalian development, the pluripotent cells of the embryo are exposed to a 16 combination of signals that drive exit from pluripotency and germ layer differentiation. At the 17 same time, a small population of pluripotent cells give rise to the primordial germ cells (PGCs),

18 the precursors of the sperm and egg, which pass on heritable genetic information to the next 19 generation. Despite the importance of PGCs, it remains unclear how they are first segregated 20 from the soma, and if this involves distinct responses to their signaling environment. To 21 investigate this question, we mapped BMP, MAPK and WNT signaling responses over time in 22 PGCs and their surrounding niche in vitro and in vivo at single-cell resolution. We showed that, 23 in the mouse embryo, early PGCs exhibit lower BMP and MAPK responses compared to 24 neighboring extraembryonic mesoderm cells, suggesting the emergence of distinct signaling 25 regulatory mechanisms in the germline versus soma. In contrast, PGCs and somatic cells 26 responded comparably to WNT, indicating that this signal alone is not sufficient to promote 27 somatic differentiation. Finally, we investigated the requirement of a BMP response for these 28 cell fate decisions. We found that cell lines with a mutation in the BMP receptor $\left(B m p r 1 a^{-/}\right)$, 29 which exhibit an impaired BMP signaling response, can efficiently generate PGC-like cells 30 revealing that canonical BMP signaling is not cell autonomously required to direct PGC-like 31 differentiation. 


\section{Introduction}

36 Primordial germ cells (PGCs) are the embryonic precursors of the sperm and egg that are

37 required to pass on heritable genetic information to the next generation. Defects in PGC

38 production result in infertility while transformed or incorrectly positioned PGCs may give rise to

39 germ cell tumors [1-4]. Thus, delineating the mechanisms that control PGC formation is critical

40 to our understanding of both development and disease.

42 In mouse, PGCs emerge during early development at a time when the pluripotent cells of the 43 embryo are exposed to a myriad of signals that drive cell fate specification. These signals direct 44 the majority of cells to adopt somatic fates [5], while a small population of only around 40 cells 45 repress the somatic program and instead become PGCs [6-8]. Despite the importance of these 46 cells, it is unclear how distinct germline and soma identities emerge within a common signaling 47 environment. Unlike somatic cells, PGCs express pluripotency-associated factors, including 48 Oct4 (Pou5f1), Sox2, Nanog, Alkaline Phosphatase, and Ssea-1 [9] and demonstrate pluripotent 49 properties, such as the capacity to give rise to self-renewing cell lines in vitro, and teratomas in 50 vivo [10,11]. This has led to the hypothesis suggesting that PGCs are the last cells of the 51 embryo to differentiate [12], and thus may not initially respond to differentiation cues. Consistent 52 with this notion, while emerging in a region of the embryo that is exposed to high levels of Bone 53 Morphogenetic Protein (BMP) signaling factor, PGCs do not exhibit a BMP signaling response 54 although their immediate somatic neighbors do [13, 14]. Nevertheless, we still know almost 55 nothing about how PGCs respond to the other biochemical signals present within their 56 environment in the embryo and how these responses change over time.

To address this, we systematically and quantitatively analyzed the response of individual PGCs

59 and neighboring somatic niche cells to key signals present within the embryo during PGC 60 specification. We confirmed that PGC-like cells (PGCLCs), generated from embryonic stem 61 cells (ESCs) in vitro, and PGCs in vivo displayed significantly lower BMP signaling responses 62 than non-PGCs. We found that early PGCs in vivo also show a diminished Mitogen-Activated 63 Protein Kinase (MAPK) response, revealing PGC-specific modes of signaling regulation for 64 multiple pathways. In contrast, PGCs responded to WNT comparably to somatic niche cells. 65 Therefore, PGCs are not refractory to all signals within their environment and, in this context, in 66 the absence of robust BMP and MAPK responses, WNT signaling is not sufficient to drive 67 somatic differentiation in cells to be assigned a PGC fate. 
69 Finally, while PGCs are devoid of a BMP signaling response, BMP is required for PGC specification [15-20], but its role and mechanism of action remain elusive. Here, we showed that

71 ESCs with a mutation in the BMP receptor type la (Bmpr1a) gene, which are defective in their

72 canonical BMP signaling response, can efficiently generate PGCLCs revealing that a robust

73 canonical BMP response is neither required transiently at earlier stages of differentiation, or

74 indirectly via the somatic niche for early PGC differentiation.

\section{Methods}

\subsection{Cell culture and PGCLC in vitro differentiation}

Cells were maintained at $37^{\circ} \mathrm{C}$, at $5 \% \mathrm{CO}_{2}$ and $90 \%$ humidity. ESC lines were routinely cultured in serum/LIF medium (Dulbecco's modified Eagle's medium (DMEM) (Gibco, Gaithersburg, MD) containing $0.1 \mathrm{mM}$ non-essential amino-acids (NEAA), $2 \mathrm{mM}$ glutamine and $1 \mathrm{mM}$ sodium pyruvate, $100 \mathrm{U} / \mathrm{ml}$ Penicillin, $100 \mu \mathrm{g} / \mathrm{ml}$ Streptomycin (all from Life Technologies, Carlsbad, CA), $0.1 \mathrm{mM}$ 2-mercaptoethanol (Sigma, St. Louis, MO), and 10\% Fetal Calf Serum (FCS, F2442, Sigma) and $1000 \mathrm{U} / \mathrm{ml}$ LIF on plates coated with $0.1 \%$ gelatin, as described [21]. The following cell lines were used in this study: E14 (129/Ola background) [22], TCF/Lef:H2B-GFP [23], Spry4 ${ }^{\mathrm{H} 2 \mathrm{~B}-\mathrm{Venus}}$ [24], and Bmpr1a-/- [25].

In vitro PGC-like cell (PGCLC) differentiation was performed as described [26]. Briefly, ESCs were converted to an epiblast-like (EpiLC) state by 48 hour culture in N2B27 medium containing 12 ng/ml FGF2 (233-FB-025, R\&D Systems) and 20 ng/ml ACTIVIN A (120-14P, Peprotech, Rocky Hills, NJ) on dishes coated with $16.7 \mu \mathrm{g} / \mathrm{mL}$ fibronectin (FC010, Millipore). Following EpiLC conversion, cells were trypsinized to a single cell suspension and 10,000 cells $/ \mathrm{mL}$ were resuspended in PGCLC medium, comprising GMEM (Gibco), 0.1 mM NEAA, 2 mM glutamine and $1 \mathrm{mM}$ sodium pyruvate, $100 \mathrm{U} / \mathrm{ml}$ Penicillin, $100 \mu \mathrm{g} / \mathrm{ml}$ Streptomycin, $0.1 \mathrm{mM} 2-$ mercaptoethanol, 1000 U/ml LIF, 15 \% Knockout serum replacement, with 500 ng/ml BMP4, $500 \mathrm{ng} / \mathrm{ml} \mathrm{BMP8a}, 100 \mathrm{ng} / \mathrm{ml} \mathrm{SCF}$, and $50 \mathrm{ng} / \mathrm{ml}$ EGF (all from R\&D Systems). Samples were collected for analysis at day 0 (EpiLC state), 2, 4 and 6 of differentiation.

\subsection{Flow cytometry}


102 Between 8-12 PGCLC aggregates per cell line/condition were pooled and then dissociated by

103 incubation in TrpLE ${ }^{\mathrm{TM}}$ Select Enzyme (Thermo Fisher Scientific) at $37^{\circ} \mathrm{C}$ for approximately 2

104 minutes. Following vigorous pipetting to form a single-cell suspension, the enzyme was

105 neutralized with an equal volume of PGCLC medium without cytokines added. Cells were

106 pelleted by centrifugation and then resuspended in $100 \mu \mathrm{L}$ FACs buffer (PBS with $10 \%$ FCS)

107 with PE-conjugated anti-CD61 (RRID:AB_313084, Biolegend, 104307, 1:200) and Alexa Fluor

108 647-conjugated anti-SSEA1 (RRID:AB_1210551, Thermo Fisher Scientific, 51-8813-73, 1:50)

109 for $15 \mathrm{~min}$ on ice. Cells were then washed in $1 \mathrm{~mL}$ FACS buffer and resuspended in $200 \mu \mathrm{L}$

110 FACS buffer containing $5 \square \mu \mathrm{g} / \mathrm{ml}$ Hoechst. Samples were analyzed using a BD LSR Fortessa ${ }^{\text {TM }}$.

111 Flow cytometry analysis was performed using FlowJo software (BD Biosciences). Cells were

112 first separated from debris and cell doublets removed by gating on forward (FSC) and side

113 scatter (SSC). Subsequently, dead cells were identified based on strong Hoechst staining and

114 were excluded from further analysis. Gating for CD61, SSEA-1 positive cells was based on

115 unstained wildtype E14 ESCs.

\subsection{Mouse lines}

119 Mice were housed under a $12 \square \mathrm{hr}$ light-dark cycle in a pathogen-free room in the designated

120 MSKCC facilities. For this study we used outbred CD1 animals maintained in accordance with

121 the guidelines of the Memorial Sloan Kettering Cancer Center (MSKCC) Institutional Animal

122 Care and Use Committee (IACUC). Natural mating was set up in the evening and mice were

123 checked for copulation plugs the next morning. The date of vaginal plug was estimated as E0.5.

124 For analysis of post-implantation stages of development, embryos were isolated from deciduae

125 and Reichert's membrane removed by microdissection before further processing.

\section{$127 \quad 2.4$ Immunostaining}

129 Cell lines were immunostained as previously described [21]. Post-implantation embryos were 130 washed in phosphate-buffered saline (PBS), then fixed in $4 \%$ paraformaldehyde (PFA) for 15 131 min at room temperature (RT). Embryos were washed in PBS plus $0.1 \%$ Triton-X (PBST-T)

132 followed by permeabilization for 30 min in PBS with $0.5 \%$ Triton-X. Embryos were then washed 133 in PBS-T and blocked overnight at $4{ }^{\circ} \mathrm{C}$ in PBS-T with $1 \%$ bovine serum albumin (BSA, Sigma) 134 and $5 \%$ donkey serum (Sigma). The following day, embryos were transferred to the primary 135 antibody solution (PBS-T with appropriate concentration of antibody) and incubated overnight at 
$1364{ }^{\circ} \mathrm{C}$. The following day, embryos were washed $3 \times 10 \mathrm{~min}$ in PBS-T and transferred to blocking 137 solution at RT for a minimum of $5 \mathrm{hr}$. Embryos were transferred to secondary antibody solution

138 (PBS-T with 1:500 dilution of appropriate secondary conjugated antibody and $5 \square \mathrm{\mu g} / \mathrm{ml} \mathrm{Hoechst)}$

139 overnight at $4{ }^{\circ} \mathrm{C}$. Embryos were washed $3 \times 10$ min in PBS-T.

141 The following primary antibodies were used in this study: AP2Y (RRID:AB_667770,

142 Santa Cruz, sc-12762, 1:100), phosphorylated SMAD1/5/9 (a gift from Dr. Edward Laufer, 143 University of Utah School of Medicine), Sox2 (RRID:AB_11219471, Thermo Fisher Scientific, 144 14-9811-82, 1:200).

\subsection{Cryosectioning}

148 Following wholemount immunostaining and imaging, embryos were oriented as desired and 149 embedded in Tissue-Tek® OCT (Sakura Finetek, Japan). Samples were frozen on dry ice for 150 approximately $30 \mathrm{~min}$ and then maintained for short periods at $-80 \square^{\circ} \mathrm{C}$ followed by 151 cryosectioning using a Leica CM3050S cryostat. Transverse cryosections of $10 \square \mu \mathrm{m}$ thickness 152 were cut with a Leica CM3050S cryostat and mounted on Colorfrost Plus $®$ microscope slides 153 (Fisher Scientific) using Fluoromount G (RRID:SCR_015961, Southern Biotech, Birmingham, $154 \mathrm{AL}$ ). Cryosections were then imaged using a confocal microscope as described.

\subsection{Quantitative image analysis}

158 Embryos were imaged on a Zeiss LSM880 laser scanning confocal microscope. Confocal z 159 stacks of cells or embryo cryosections were generated. Raw data was then processed in 160 ImageJ open source image processing software (Version: 2.0.0-rc-49/1.51d). Individual 161 PGCLCs, identified by AP2y expression, PGCs identified by SOX2 expression, or their 162 surrounding AP2y-SOX2- niche cells were randomly chosen and, using Fiji (ImageJ) software, 163 selected by manually drawing a boundary around the nucleus. The mean fluorescence intensity 164 of pSMAD1/5/9 immunostaining, Spry4 $4^{\text {H2B-Venus }}$, or TCF/Lef:H2B-GFP reporter expression was 165 then measured in arbitrary units. Fluorescence decay along the z-axis was corrected for each 166 channel and sample by fitting a linear regression model to the logarithm of fluorescence values

167 as a function of the z-value, and correcting the models' slopes using an empirical Bayes 168 approach, as previously described [27]. For all quantification, statistical analysis of significance 169 was assessed using a One-way ANOVA followed by unpaired $t$-tests to compare particular 
170 groups (GraphPad Prism, GraphPad Software, Inc., Version 7.0a). For analysis performed on

171 embryos, all PGCs were selected from 3 different cryosections through the allantois of 3 distinct

172 embryos. Fluorescence values were then calculated relative to the average mean fluorescence

173 of non-neighboring ('Other') AP2y-SOX2- niche cells within each individual section in order to

174 normalize for differences in immunostaining that may arise due to differences in permeability

175 within different embryonic regions or different stages of development. Statistics were carried out

176 on average fluorescence levels per embryo, rather than on a per cell basis.

177

\section{Results and discussion}

\subsection{Quantitative analysis of signaling responses during mouse PGCLC specification}

Functional PGC-like cells (PGCLCs) can be generated in vitro from mouse embryonic stem cells (ESCs). First ESCs are converted to an epiblast-like cell (EpiLC) state, comparable to the pluripotent embryonic cells before germ layer differentiation. Subsequently, EpiLCs are aggregated in suspension culture and exposed to a combination of signals, mimicking those present in the embryo, that promote PGC specification, survival, and proliferation (Fig. 1A) [26]. Using this protocol, we successfully generated PGCLCs, identified by the coexpression of SOX2 and AP2y (Fig. 1B), and the cell surface markers SSEA-1 and CD61 (Fig. 1C, D) [28]. PGCLC aggregates displayed widespread SOX2 expression while AP2 $y$ was expressed in only a subset of cells, suggesting that the rate of PGCLC specification was variable across individual cells or that a mixture of cell fates were formed (Fig. 1B). Thus, we considered PGCLCs as cells that coexpressed SOX2 and AP2y in our downstream analyses. Using this cell culture system, we then analyzed signaling responses in individual PGCLCs and surrounding non-PGCLCs.

195 BMP signaling plays a critical role in PGC specification. Mutations in the genes encoding Bmp4, 196 Bmp8, and Bmp2, as well as the downstream effectors that mediate the BMP signaling 197 response, Smad1 and Smad5, result in a loss or significant reduction in PGC number [15-20]. 198 Nevertheless, neither PGCLCs in vitro nor PGCs in vivo exhibit a canonical BMP signaling 199 response $[13,14]$, demonstrated by the absence of nuclear-localized phosphorylated 200 SMAD1/5/9 (pSMAD1/5/9, SMAD9 is also known as SMAD8). However, BMP responses have 201 not been systematically and quantitatively analyzed at single-cell resolution and therefore it is 202 unclear whether a fraction of PGCs do respond or if a transient response may occur. To 203 investigate this, we measured pSMAD1/5/9 levels in individual nuclei within PGCLC aggregates 
204 at days 2, 4, and 6 of differentiation. We observed that AP2 $y+$ PGCLCs displayed significantly 205 lower levels of nuclear pSMAD1/5/9 than AP2y negative (AP2y-) non-PGCLCs (Fig. 1E, F). 206 Indeed, we did not identify any PGCLCs with nuclear-localized pSMAD1/5/9 (Fig. 1E, F). 207 Furthermore, while the BMP signaling response increased in AP2 $\gamma$ - non-PGCLCs over time, it 208 remained low in PGCLCs (Fig. 1F). Thus, at this resolution, we observed no evidence for a 209 subset of BMP-responsive PGCLCs.

We then asked whether PGCLCs also lack responses to other critical signals present within the 212 mouse embryo at this time. FGF is expressed within the posterior of the embryo at the time of 213 PGC specification and is necessary for somatic germ layer specification, gastrulation EMT and 214 concomitant cell migration [29-31]. Additionally, FGF and EGF, which both activate the MAPK 215 pathway, are provided exogenously during PGCLC differentiation (Fig. 1A). In order to analyze 216 the MAPK signaling response, we used a Spry $4^{\mathrm{H} 2 \mathrm{BV} \text { enus }}$ ESC line, which harbors a fluorescent 217 reporter in the endogenous locus of Sprouty4 (Spry4), an early target of the pathway [24]. We 218 observed widespread Venus expression throughout PGCLC aggregates at all stages of 219 differentiation (Fig. 2G). We then performed flow cytometry and quantitative 220 immunofluorescence to determine how this response changed over time. In contrast to the 221 gradually increasing BMP response in non-PGCLCs, there was a reduction in the MAPK 222 response over time (Fig. 1H, I). Quantitative immunofluorescence revealed no significant 223 difference in the MAPK signaling response in PGCLCs and non-PGCLCs (Fig. 1I). At each 224 stage of differentiation, Venus levels were lower, although not significantly, in AP2 $\gamma+$ vs. AP2 $\gamma^{-}$ 225 cells (Fig. 1I).

227 WNT signaling is required for both somatic [32-35] and germ cell [36, 37] fate specification.

228 WNT drives the initial exit from pluripotency but a subset of its targets must subsequently be 229 repressed in PGC-fated cells to prevent somatic differentiation [37]. Here we used a

230 TCF/Lef:H2B-GFP reporter ESC line, that contains multimerized binding sides for the T cell-

231 specific transcription factor/lymphoid enhancer-binding factor 1 (TCF/Lef) family of transcription

232 factor coactivators, which mediate the WNT signaling response [23]. Although recombinant

233 WNT is not added exogenously to the PGCLC differentiation medium, TCF/Lef:H2B-GFP was

234 expressed heterogeneously throughout cell aggregates (Fig. 1J), signifying that endogenous

235 WNT ligands were present. However, there was no difference in the WNT response in PGCLCs

236 compared to non-PGCLCs revealing that PGCLCs are not refractory to all differentiation-

237 inducing signals. The WNT response decreased during PGCLC differentiation (Fig. 1K, L). 
238 Thus, initially PGCLCs show a reduced BMP signaling response and as differentiation

239 proceeds, PGCLCs and non-PGCLCs also reduce their MAPK and WNT signaling responses.

\subsection{Quantitative analysis of signaling responses during PGC specification in vivo}

The combination, dynamics, and dose of factors provided during PGCLC differentiation in vitro,

244 may not precisely recapitulate the dynamic signaling environment within the embryo. Moreover,

245 the majority of AP2y- non-PGCLCs also expressed SOX2 (Fig. 1B), suggesting that they

246 represent a pluripotent EpiLC state or earlier state of PGCLC differentiation, and thus do not

247 mirror the in vivo PGC niche at the posterior of the embryo that comprises extraembryonic

248 mesoderm. Therefore, we also sought to investigate signaling responses in PGCs and their

249 niche in the embryo. We isolated and analyzed embryos at embryonic day (E) 7.25, when

250 SOX2 + AP2 $\gamma+$ PGCs first emerge within a posteriorly-localized extraembryonic structure known

251 as the allantois (Fig. 2A) [38], and at E7.75, when PGCs begin to exit the allantois and migrate

252 anteriorly along the hindgut endoderm toward their eventual destination in the gonads. In

253 contrast to PGCLC aggregates, where only a subset of SOX2+ cells expressed AP2y, in vivo

254 SOX2 and AP2y expression fully overlapped (Fig. 2A). However, as AP2y immunofluorescence

255 resulted in high levels of non-specific background staining in the endoderm on the embryo's

256 surface (Fig. 2A), we used SOX2 to accurately identify PGCs. We isolated wildtype embryos,

257 which we immunostained for pSMAD1/5/9, as well as Spry4 ${ }^{\text {H2B-Venus }}$, and TCF/Lef:H2B-GFP

258 reporter embryos and measured signaling responses in individual SOX2+ PGCs, and SOX2-

259 non-PGCs that were either adjacent to PGCs (categorized as 'Neighbors'), or non-adjacent

260 (categorized as 'Other') in transverse cryosections of the allantois (Fig. 2A, B, C). As in

261 PGCLCs, PGCs at E7.25 and E7.75 showed significantly lower levels of nuclear-localized

262 pSMAD1/5/9 than both neighboring and non-neighboring SOX2- cells within the surrounding

263 somatic niche (Fig. 2D, E).

265 Cells within the allantois showed widespread Spry $4^{\mathrm{H} 2 \mathrm{BV} \text { enus }}$ expression (Fig. 2F). However, at

266 E7.25, PGCs displayed a significantly reduced MAPK response compared to non-PGC 267 neighbors and non-neighboring SOX2- cells (Fig. 2F, G). By E7.75, this difference was no 268 longer significant (Fig. 2G). PGCs within the hindgut endoderm displayed a higher MAPK 269 response than their PGC counterparts within the allantois (Fig. 2G). Moreover, the MAPK 270 response was higher in endoderm relative to extraembryonic mesoderm (Fig. 2G). Therefore, as 
271 PGCs migrate towards the gonads, they enter an environment of higher MAPK signaling

272 activity.

274 During PGCLC differentiation there was no difference in the WNT response in PGCLCs vs. non-

275 PGCLCs (Fig. 1L). In contrast, at E7.25 in vivo, PGCs expressed higher levels of TCF/Lef:H2B-

276 GFP than non-adjacent extraembryonic mesoderm cells (Fig. 2H, I), likely a result of the distinct

277 nature of the in vitro and in vivo niches. By E7.75, there was no difference in the WNT response

278 between PGCs and their neighbors. However, migrating PGCs exhibited a stronger WNT

279 response than non-adjacent endoderm. Thus, both in vitro and in vivo, PGCs respond to WNT.

3.3 BMP signaling response is not required for PGCLC specification

While BMP is required for PGC specification [15-20], and BMP4 and BMP8a (500 ng/UL) are exogenously provided during PGCLC differentiation [26], we and others observed that neither PGCLCs or PGCs exhibit nuclear-localized pSMAD1/5/9 (Fig. 1E, F, 2C, D) [13, 14]. Thus, either a transient BMP response is required for PGC specification that we do not capture at this temporal resolution, or BMP is required indirectly by PGCs. To distinguish between these possibilities, we performed PGCLC differentiation of Bmpr1a ${ }^{-/}$ESCs [25]. Bmpr1a is the most broadly and highly expressed BMP receptor within the pluripotent epiblast during PGC specification [39] and Bmpr1a ${ }^{-/}$embryos exhibit little or no nuclear pSMAD1/5/9 [40]. In keeping with this, and as previously observed [25], Bmpr1a ${ }^{-/}$ESCs did not display nuclear-localized pSMAD1/5/9 under standard serum/LIF culture conditions, although this was observed in wildtype Bmpr1a ${ }^{+/+}$ESCs (Fig. 3A), or when treated with BMP4 for 2 hours (Fig. 3B).

294 Comparable observations were made with Bmpr1a ${ }^{-/-}$EpiLCs (Fig. 3C). We then exposed 295 Bmpr1a $^{-/-}$EpiLCs to PGCLC induction medium and showed that, likewise, Bmpr1a ${ }^{-/}$cell 296 aggregates do not exhibit nuclear-localized pSMAD1/5/9 under these conditions (Fig. 3D, E).

298 Despite this, we observed the specification of cells that expressed AP2 $y$, as well as SSEA-1 and 299 CD61 (Fig. 3D, F, G). Notably, Bmpr1a ${ }^{-/}$EpiLCs showed a slightly higher percentage of SSEA$3001+$ CD61 + cells than wildtype EpiLCs prior to exposure to PGCLC medium, and accordingly 301 they displayed an earlier peak in this population during differentiation (Fig. 3G). This finding 302 suggested that cells with a low BMP response could be predisposed towards a PGCLC fate. 303 Consistent with this notion, we also noted an inverse correlation between the expression of the 304 BMP pathway target Inhibitor of differentiation 1 (ID1) and the PGC marker AP2y in wildtype 
305 ESCs (Fig. 3H). To investigate this further, we then mixed equal proportions of $B m p r 1 a^{+/+}$and

306 Bmpr1a ${ }^{-/}$EpiLCs, to form chimeric PGCLC aggregates (Fig. 3I). Bmpr1a ${ }^{-/}$ESCs were lineage

307 labelled with a constitutive GFP reporter that enabled tracking of their eventual fate. In these

308 experiments, we observed variable proportions of GFP+ cells within the resulting aggregates

309 (data not shown). In aggregates with a low percentage of GFP+ cells, it was predominantly

310 Bmpr1a $^{-/-}$cells that gave rise to and were immediately adjacent to AP2Y+ PGCLCs (Fig. 3I).

311 Together these data indicate that a canonical BMP response is not required cell autonomously

312 for PGCLC differentiation.

\section{Discussion}

316 While many of the signals that direct PGC specification have been elucidated [14, 36, 41], little

317 is known about how individual PGCs and their niche respond to these signals and whether their

318 role is cell-autonomous or non-cell-autonomous. BMP is required for PGC development [15-20].

319 While BMP8b and BMP2 act non-cell autonomously to restrict the development of the anterior

320 visceral endoderm, a source of PGC inhibitory signals, and to specify the hindgut required for

321 PGC migration respectively [20,36], the role of BMP4 is still unclear. Our and other

322 observations $[13,14]$ that PGCs do not exhibit a canonical BMP signaling response, suggest

323 that BMP4 likewise acts non-cell-autonomously or that low-levels of BMP signaling activity, not

324 detectable following antibody staining, could be sufficient for PGC specification. This is

325 supported by our finding that BMP signaling defective $\left(B m p r 1 a^{-/}\right)$ESCs efficiently give rise to

326 PGCLCs. However, as Bmpr1a ${ }^{-/-}$PGCLC differentiation occurred in the absence of wildtype

327 cells, the requirement for BMP is not via BMP-responsive cells within the niche and may instead

328 be through non-canonical SMAD-independent downstream pathways [42, 43]. Alternatively, as

329 perturbation of BMP signaling in vivo causes the epiblast to prematurely adopt a neural identity

330 [25], it may be required to initially maintain the epiblast in a PGC competent state rather than

331 directly regulating PGC specification. This role may not be masked in vitro as ESCs are forcibly

332 maintained in a self-renewing state using $2 \mathrm{i}$ small molecule inhibitors.

334 MAPK inhibition promotes PGC differentiation in vitro [44]. Additionally, treating isolated PGCs

335 with FGF reprograms them to a state of pluripotency [45]. Thus, FGF/MAPK signaling is

336 associated with a block to the formation, or destabilization, of a PGC identity. In keeping with

337 this, we observed that early PGCs, at E7.25, showed a lower MAPK response than somatic

338 cells. Although we observed low-level Spry $4^{\text {H2BVenus }}$ expression within PGCs and PGCLCs, 
339 indicating that MAPK signaling may not be entirely shut down, this could also represent 340 perdurance of the Venus reporter. Therefore, future studies using dynamic ERK biosensors [46, 341 47], combined with time-lapse imaging [48], will uncover precise signaling dynamics. We also

342 observed that the MAPK response was elevated in PGCs within the hindgut endoderm, 343 consistent with studies showing that FGF plays a role in germ cell migration [45, 49]. This is at 344 odds with reports that migrating PGCs are devoid of phosphorylated ERK, a component of the 345 MAPK pathway [8]. Therefore, Spry4 expression within the endoderm may also be regulated by 346 additional signaling inputs, such as WNT [50].

348 PGCs are specified in a signaling environment that instructs the majority of cells to adopt a somatic non-PGC identity. One way that they might maintain their unique identity is via 350 regulatory mechanisms that prevent them from detecting or responding to these signals. 351 Nevertheless, while PGCs displayed a reduced BMP and MAPK response, they did respond to 352 WNT. Hence, in the absence of robust BMP and MAPK responses, WNT is not sufficient to 353 drive somatic differentiation. Previous studies reported that, after an initial period of WNT 354 signaling, WNT targets must be suppressed to mediate PGC differentiation [37]. Consistent with 355 this, we observed a rapid decrease in the WNT signaling response over time during PGCLC 356 differentiation. However, PGCs and somatic cells showed comparable levels of TCF/Lef:H2B357 GFP expression suggesting that there is not a global PGC-specific reduction in the signaling 358 response. Thus, this regulation likely occurs via locus-specific mechanisms [37]. Of cells within 359 the allantois, PGCs exhibit the strongest WNT response, followed by their immediate somatic 360 neighbors, while non-neighboring somatic cells were the least responsive. Therefore, PGCs 361 could perhaps act as a source of WNT that activates autocrine and paracrine signaling in 362 adjacent, but not more distant cells.

364 Here we have shown that PGC-specific signaling responses exist for a number of different 365 pathways. However, while we observed significant differences in MAPK and WNT signaling 366 responses in PGCs vs. somatic cells within the allantois, these were not evident in PGCLC 367 aggregates. This presumably reflects the difference between the PGC niche in the embryo and 368 during stem cell differentiation, highlighting the importance of side by side comparisons of in 369 vitro and in vivo developmental mechanisms. Furthermore, the important question remains as to 370 how these distinct PGCs and soma responses are regulated. Current single-cell transcriptomic 371 studies of mouse embryos contain only a small number of PGCs with no spatial information, 372 thereby prohibiting clear conclusions on the relative expression of signaling pathway 
373 components within these cells versus their immediate neighbors and cells within their further

374 proximity. Future PGC-enriched single-cell spatial transcriptomic studies may shed light on this.

375 However, as signaling responses are largely regulated at a post-transcriptional level, advances

376 in single-cell proteomic techniques or the use of quantitative time and space resolved reporters

377 as dynamic signaling readouts may be necessary to fully answer these open questions.

\section{Acknowledgements}

381 We thank members of the Hadjantonakis lab for critical discussions and comments on the

382 manuscript. We also thank members of MSKCC's Flow Cytometry Core facility, funded by the

383 NCl Cancer Center Support Grant (CCSG, P30 CA08748). Additionally, we thank Tristan

384 Rodriguez for providing the Bmpr1a ${ }^{-/}$ESCs used within this study. SMM was supported by a

385 Wellcome Trust Sir Henry Wellcome postdoctoral fellowship under the supervision of AKH.

386 Work in the Hadjantonakis lab was supported by grants from the NIH (R01HD094868,

387 R01DK084391 and P30CA008748).

\section{Ethics}

390 Animal experimentation: Animal experimentation: All mice used in this study were maintained in 391 accordance with the guidelines of the Memorial Sloan Kettering Cancer Center (MSKCC)

392 Institutional Animal Care and Use Committee (IACUC) under protocol number 03-12-017 (PI

393 Hadjantonakis). 


\section{References}

398 1. Pierce, J.L., A.L. Frazier, and J.F. Amatruda, Pediatric Germ Cell Tumors: A Developmental $399 \quad$ Perspective. Advances in Urology, 2018. 2018.

$400 \quad 2 . \quad$ Stevens, L.C., The biology of teratomas. Adv Morphog, 1967. 6: p. 1-31.

$4013 . \quad$ Stevens, L.C., Teratocarcinogenesis and spontaneous parthenogenesis in mice. Results Probl Cell Differ, 1980. 11: p. 265-74.

4. Giuliano, C.J., S.J. Freemantle, and M.J. Spinella, Testicular Germ Cell Tumors: A Paradigm for the Successful Treatment of Solid Tumor Stem Cells. Curr Cancer Ther Rev, 2006. 2(3): p. 255-270.

5. Morgani, S.M. and A.K. Hadjantonakis, Signaling regulation during gastrulation: Insights from mouse embryos and in vitro systems. Gradients and Tissue Patterning, 2020. 137: p. 391-431.

6. Magnusdottir, E., et al., A tripartite transcription factor network regulates primordial germ cell specification in mice. Nature Cell Biology, 2013. 15(8): p. 905-U322.

7. Ohinata, Y., et al., Blimp1 is a critical determinant of the germ cell lineage in mice. Nature, 2005. 436(7048): p. 207-213.

8. Grabole, N., et al., Prdm14 promotes germline fate and naive pluripotency by repressing FGF signalling and DNA methylation. EMBO Rep, 2013. 14(7): p. 629-37.

9. Irie, N., W.W. Tang, and M. Azim Surani, Germ cell specification and pluripotency in mammals: a perspective from early embryogenesis. Reprod Med Biol, 2014. 13(4): p. 203-215.

10. Matsui, Y., K. Zsebo, and B.L.M. Hogan, Derivation of Pluripotential Embryonic Stem-Cells from Murine Primordial Germ-Cells in Culture. Cell, 1992. 70(5): p. 841-847.

11. Resnick, J.L., et al., Long-Term Proliferation of Mouse Primordial Germ-Cells in Culture. Nature, 1992. 359(6395): p. 550-551.

12. Johnson, A.D. and R. Alberio, Primordial germ cells: the first cell lineage or the last cells standing? Development, 2015. 142(16): p. 2730-2739.

13. Dudley, B.M., et al., BMP signaling regulates $P G C$ numbers and motility in organ culture. Mechanisms of Development, 2007. 124(1): p. 68-77.

14. Senft, A.D., et al., Genetic dissection of Nodal and Bmp signalling requirements during primordial germ cell development in mouse. Nature Communications, 2019. 10.

15. Chang, H. and M.M. Matzuk, Smad5 is required for mouse primordial germ cell development. Mechanisms of Development, 2001. 104(1-2): p. 61-67.

16. Hayashi, K., et al., SMAD1 signaling is critical for initial commitment of germ cell lineage from mouse epiblast. Mechanisms of Development, 2002. 118(1-2): p. 99-109.

17. Lawson, K.A., et al., Bmp4 is required for the generation of primordial germ cells in the mouse embryo. Genes \& Development, 1999. 13(4): p. 424-436.

18. Tremblay, K.D., N.R. Dunn, and E.J. Robertson, Mouse embryos lacking Smad1 signals display defects in extra-embryonic tissues and germ cell formation. Development, 2001. 128(18): p. 3609-3621.

19. Ying, Y., et al., Requirement of Bmp8b for the generation of primordial germ cells in the 
20. Ying, Y. and G.Q. Zhao, Cooperation of endoderm-derived BMP2 and extraembryonic ectoderm-derived BMP4 in primordial germ cell generation in the mouse. Developmental Biology, 2001. 232(2): p. 484-492.

21. Morgani, S.M., et al., Micropattern differentiation of mouse pluripotent stem cells recapitulates embryo regionalized cell fate patterning. Elife, 2018. 7.

22. Hooper, M., et al., HPRT-deficient (Lesch-Nyhan) mouse embryos derived from germline colonization by cultured cells. Nature, 1987. 326(6110): p. 292-5.

23. Ferrer-Vaquer, A., et al., A sensitive and bright single-cell resolution live imaging reporter of Wnt/ss-catenin signaling in the mouse. BMC Dev Biol, 2010. 10: p. 121.

24. Morgani, S.M., et al., A Sprouty4 reporter to monitor FGF/ERK signaling activity in ESCS and mice. Developmental Biology, 2018. 441(1): p. 104-126.

25. Di-Gregorio, A., et al., BMP signalling inhibits premature neural differentiation in the mouse embryo. Development, 2007. 134(18): p. 3359-3369.

26. Hayashi, K., et al., Reconstitution of the mouse germ cell specification pathway in culture by pluripotent stem cells. Cell, 2011. 146(4): p. 519-32.

27. Saiz, N., et al., Asynchronous fate decisions by single cells collectively ensure consistent lineage composition in the mouse blastocyst. Nature Communications, 2016. 7.

28. Hayashi, K. and M. Saitou, Stepwise Differentiation from Naive State Pluripotent Stem Cells to Functional Primordial Germ Cells Through an Epiblast-Like State. Epiblast Stem Cells: Methods and Protocols, 2013. 1074: p. 175-183.

29. Yamaguchi, T.P., et al., Fgfr-1 Is Required for Embryonic Growth and Mesodermal Patterning during Mouse Gastrulation. Genes \& Development, 1994. 8(24): p. 30323044.

30. Ciruna, B. and J. Rossant, FGF signaling regulates mesoderm cell fate specification and

31. Deng, C.X., et al., Murine Fgfr-1 Is Required for Early Postimplantation Growth and Axial

32. Barrow, J.R., et al., Wnt3 signaling in the epiblast is required for proper orientation of the anteroposterior axis. Developmental Biology, 2007. 312(1): p. 312-320.

33. Liu, P.T., et al., Requirement for Wnt3 in vertebrate axis formation. Nature Genetics, 1999. 22(4): p. 361-365.

34. Haegel, $\mathrm{H}_{\text {., }}$ et al., Lack of beta-catenin affects mouse development at gastrulation. Development, 1995. 121(11): p. 3529-37.

35. Kelly, O.G., K.I. Pinson, and W.C. Skarnes, The Wnt co-receptors Lrp5 and Lrp6 are essential for gastrulation in mice. Development, 2004. 131(12): p. 2803-2815.

36. Ohinata, Y., et al., A Signaling Principle for the Specification of the Germ Cell Lineage in Mice. Cell, 2009. 137(3): p. 571-584.

37. Aramaki, S., et al., A Mesodermal Factor, T, Specifies Mouse Germ Cell Fate by Directly Activating Germline Determinants. Developmental Cell, 2013. 27(5): p. 516-529.

38. Ginsburg, M., M.H.L. Snow, and A. Mclaren, Primordial Germ-Cells in the Mouse Embryo during Gastrulation. Development, 1990. 110(2): p. 521-\&.

39. Pijuan-Sala, B., et al., A single-cell molecular map of mouse gastrulation and early organogenesis. Nature, 2019. 566(7745): p. 490-+. 
40. Mishina, Y., et al., Bmpr encodes a type I bone morphogenetic protein receptor that is essential for gastrulation during mouse embryogenesis. Genes \& Development, 1995. 9(24): p. 3027-3037.

41. Saitou, M. and M. Yamaji, Germ cell specification in mice: signaling, transcription regulation, and epigenetic consequences. Reproduction, 2010. 139(6): p. 931-942.

42. Derynck, R. and Y.E. Zhang, Smad-dependent and Smad-independent pathways in TGFbeta family signalling. Nature, 2003. 425(6958): p. 577-84.

43. Zhang, Y.E., Non-Smad pathways in TGF-beta signaling. Cell Res, 2009. 19(1): p. 128-39.

44. Kimura, T., et al., Induction of Primordial Germ Cell-Like Cells From Mouse Embryonic Stem Cells by ERK Signal Inhibition. Stem Cells, 2014. 32(10): p. 2668-2678.

45. Chang, C.T., et al., Cell-intrinsic Fgf signaling contributes to primordial germ cell homing in zebrafish. Theriogenology, 2020. 158: p. 424-431.

46. Pokrass, M.J., et al., Cell-Cycle-Dependent ERK Signaling Dynamics Direct Fate Specification in the Mammalian Preimplantation Embryo. Developmental Cell, 2020. 55(3): p. 328-+.

47. Simon, C.S., et al., Live Visualization of ERK Activity in the Mouse Blastocyst Reveals Lineage-Specific Signaling Dynamics. Developmental Cell, 2020. 55(3): p. 341-+.

48. McDole, K., et al., In Toto Imaging and Reconstruction of Post-Implantation Mouse

49. Takeuchi, Y., et al., The roles of FGF signaling in germ cell migration in the mouse. Development, 2005. 132(24): p. 5399-409.

50. Katoh, Y. and M. Katoh, FGF signaling inhibitor, SPRY4, is evolutionarily conserved target of WNT signaling pathway in progenitor cells. International Journal of Molecular 
Figure 1. Quantitative analysis of signaling responses during PGCLC differentiation. A.

514 Schematic diagram depicting the PGCLC differentiation protocol as previously described [26].

515 B. Confocal maximum intensity projection of an aggregate of cells at Day 2 (D2) of PGCLC

516 differentiation. Scale bars, $100 \mu \mathrm{m}$. C. Representative flow cytometry data of embryonic stem

517 cells during PGCLC differentiation. SSEA-1 and CD61 double positive cells mark PGCLCs. D.

518 Percentage of SSEA-1 + CD61+ PGCLCs over time during PGCLC differentiation. Each point

519 represents an independent experiment $(n=6)$ performed with 4 distinct cell lines. Data

520 represented as median and interquartile range. E, G, J. Confocal maximum intensity projections

521 of PGCLC aggregates at day 2, 4, and 6 of differentiation. Scale bars, $100 \mu \mathrm{m}$. E. Aggregates

522 were immunostained for and AP2y to mark PGCLCs and phosphorylated SMAD1/5/9 (pS1/5/9),

523 a readout of the BMP signaling response. G. PGLC differentiation of Spry $4^{\mathrm{H} 2 \mathrm{BV} \text { enus }}$ reporter

524 embryonic stem cell lines, that act as a read out of FGF/MAPK signaling activity. J. PGLC

525 differentiation of TCF/Lef:H2B-GFP reporter embryonic stem cell lines, which act as a read out

526 of WNT signaling activity. F, I, L. Quantitative immunofluorescence analysis of signaling

527 responses, measured in arbitrary units (a.u.), in PGCLCs (AP2 $\gamma+$ ) and non-PGCLCs (AP2 $\gamma-)$ in

5283 distinct cell aggregates per time point per cell line. Each point represents a single cell. Data

529 shown as median and interquartile range. Statistical analysis of significance was assessed on

530 log-normalized data using Student's $t$-test, performed on the average fluorescence level in each

531 aggregate. $\mathbf{H}, \mathbf{K}$. Relative Spry4 ${ }^{\mathrm{H} 2 \mathrm{BV} \text { enus }}(\mathrm{H})$ and TCF/Lef:H2B-GFP $(\mathrm{K})$ fluorescence levels in

532 arbitrary units (a.u.) analyzed by flow cytometry in SSEA-1+ CD61+ PGCLCs, and SSEA-1-

533 CD61+, SSEA-1+ CD61- and SSEA-1 CD61- non-PGCLC populations. Data represented as

534 mean and standard deviation and shown relative to the mean fluorescence across all

535 populations at day 0 of differentiation, $n=3$ independent experiments.

Figure 2. Quantitative analysis of signaling responses during PGC specification in vivo.

538 A. (i) Sagittal confocal optical section of an immunostained embryonic day (E) 7.25 embryo.

539 Scale bar, $100 \mu \mathrm{m}$. Dashed line indicates the plane of transverse section shown in adjacent

540 panel. (ii) Confocal optical section of a transverse cryosection through the allantois of an E7.25

541 embryo. Scale bar, $25 \mu \mathrm{m}$. Box demarcates the region shown in higher magnification in lower

542 panels. AP2y immunostaining exhibits high levels of non-specific background staining within the

543 endoderm. B. For quantitative analysis of signaling responses, cells adjacent to PGCs

544 (pseudocolored in yellow) were categorized as PGC 'Neighbors' and non-adjacent cells within 
545 the allantois (pseudocolored in blue) were categorized as 'Other'. C. Quantification of levels of

546 SOX2 in arbitrary units (a.u.) PGCs, PGC Neighbors and Other cells within the allantois of

547 E7.25 embryos. SOX2+ immunostaining was used to define the PGC population. Statistical

548 analysis of significance was assessed using Student's $t$-test and performed on the average

549 fluorescence level in each embryo ( $n=3$ embryos, number of individual cells shown on graph).

550 Each point represents a single cell. Data shown relative to the average mean fluorescence in

551 'Other', non-PGCs and represented as the median and interquartile range. D, F, H. Sagittal

552 confocal maximum intensity projections (left panels, scale bars, $100 \mu \mathrm{m}$ ) and confocal optical

553 sections of transverse cryosection through the allantois of E7.25 and E7.75 embryos (scale

554 bars, $25 \mu \mathrm{m})$. Dashed line approximately demarcates the boundary between the allantois and

555 the endoderm. D. Embryos were immunostained for phosphorylated SMAD1/5/9 as a readout of

556 BMP signaling activity. F. Transgenic Spry $4^{\mathrm{H} 2 \mathrm{BV} \text { enus }}$ reporter embryos were used to read out

557 FGF/MAPK signaling activity. H. TCF/Lef:H2B-GFP reporter embryos were used to read out

558 WNT signaling activity. E, G, I. Quantification of levels of nuclear SMAD1/5/9, Spry4 ${ }^{\text {H2BVenus }}$, and

559 TCF/Lef:H2B-GFP expression in arbitrary units (a.u.) in PGCs, PGC Neighbors and Other cells

560 within the allantois of E7.25 and E7.75 embryos. In E7.25 embryos, all PGCs were within the

561 allantois. In E7.75 embryos, a fraction of PGCs had also begun to migrate along the hindgut

562 endoderm hence we separately investigated signaling responses in PGCs within the allantois

563 and within the endoderm for this analysis. Statistical analysis of significance was assessed

564 using Student's $t$-test and performed on the average fluorescence level in each embryo $(n=3$

565 embryos, number of individual cells shown on graph). Each point represents a single cell. Data

566 shown relative to the average mean fluorescence in 'Other', non-PGCs and represented as the

567 median and interquartile range. Pr, proximal; Ds, distal; A, anterior; P, posterior; L, left; R, right;

568 Epi, epiblast; PS, primitive streak; End, endoderm.

Figure 3. Cell autonomous BMP signaling response is not necessary for PGCLC fate. A.

571 Confocal optical sections of wildtype $\left(B m p r 1 a^{+/+}\right)$and Bmpr1a ${ }^{-/-}$embryonic stem cells (ESCs)

572 immunostained for pSMAD1/5/9 (pS1/5/9) after culture under standard or after 2 hours

573 treatment with $50 \mathrm{ng} / \mathrm{ml}$ BMP4. B, C. Quantification of pSMAD1/5/9 levels in wildtype and

574 Bmpr1a ${ }^{-/}$embryonic stem cells ESCs (from 5 distinct fields of view) and EpiLCs (from 5 distinct

575 fields of view). Each point represents a single cell. Data represented as median and interquartile

576 range. Statistical analysis of significance was assessed using Student's $t$-test, performed on the

577 average fluorescence level in each field. $n=2$ experimental replicates. $D$. Confocal maximum

578 intensity projection of wildtype and $\mathrm{Bmpr}^{-\mathrm{a}^{-/}}$cell aggregates at Day 2 (D2) of PGCLC 
579 differentiation. Scale bars, $100 \mu \mathrm{m}$. E. Quantification of pSMAD1/5/9 levels in wildtype and

580 Bmpr1a ${ }^{-/-}$PGCLC aggregates. Each point represents a single cell. Data represented as median

581 and interquartile range. Statistical analysis of significance was assessed using Student's $t$-test

582 and performed on the average fluorescence level in each aggregate ( $n=3$ aggregates). F. Flow

583 cytometry analysis of wildtype and Bmpr1a ${ }^{-/}$cell aggregates at Day 2 of PGCLC differentiation.

584 SSEA-1+ CD61+ cells represent PGCLCs. G. Percentage of SSEA-1+ CD61+ PGCLCs during

585 wildtype and Bmpr1a/- PGCLC differentiation. Each point represents an independent

586 experiment $(n=3)$. Data represented as median and interquartile range. $\mathbf{H}$. Confocal optical

587 section of ESCs, cultured in serum and LIF, immunostained for the BMP pathway target, ID1

588 and the PGC marker AP2y (left panel). Scale bar, $25 \mu \mathrm{m}$. Right panel shows quantification of

589 ID1 and AP2y levels in arbitrary units (a.u.) in individual cells. Quantification performed on

590 images from 5 randomly selected regions. Each point represents a single cell. Linear regression

591 and correlation coefficient analysis were performed and were statistically significant $(p<0.0001)$.

592 Correlation coefficient is shown on graph. I. Wildtype and Bmpr1a ${ }^{-/-}$EpiLCs were mixed

593 together in equal ratios to form PGCLC aggregates. Bmpr1a $a^{-/-}$cells were labelled with a

594 constitutive GFP lineage marker. Images show Confocal maximum intensity projections of

595 PGCLC aggregates at day 2, 4, and 6 of differentiation. Scale bars, $100 \mu \mathrm{m}$. 

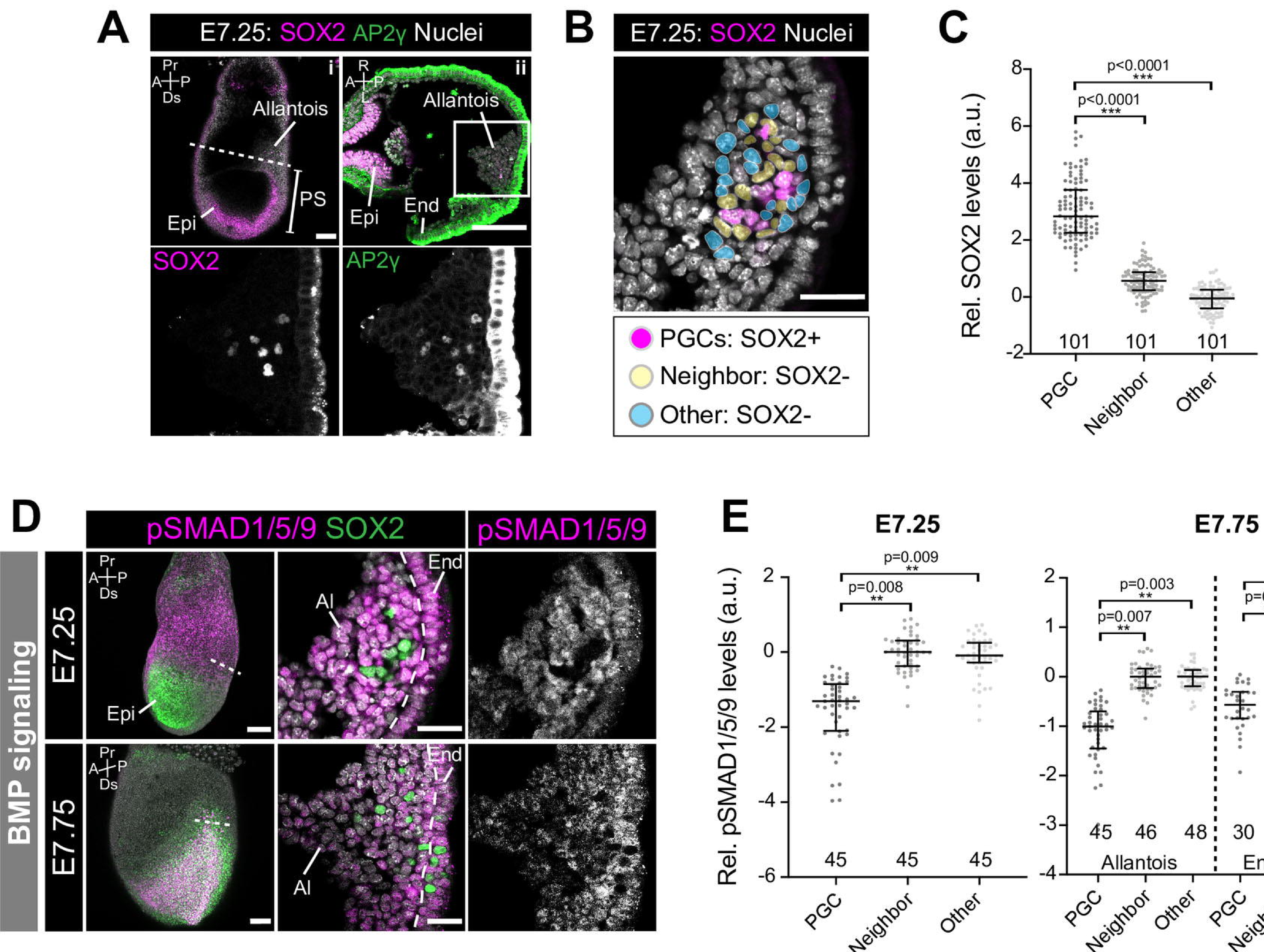

E7.75
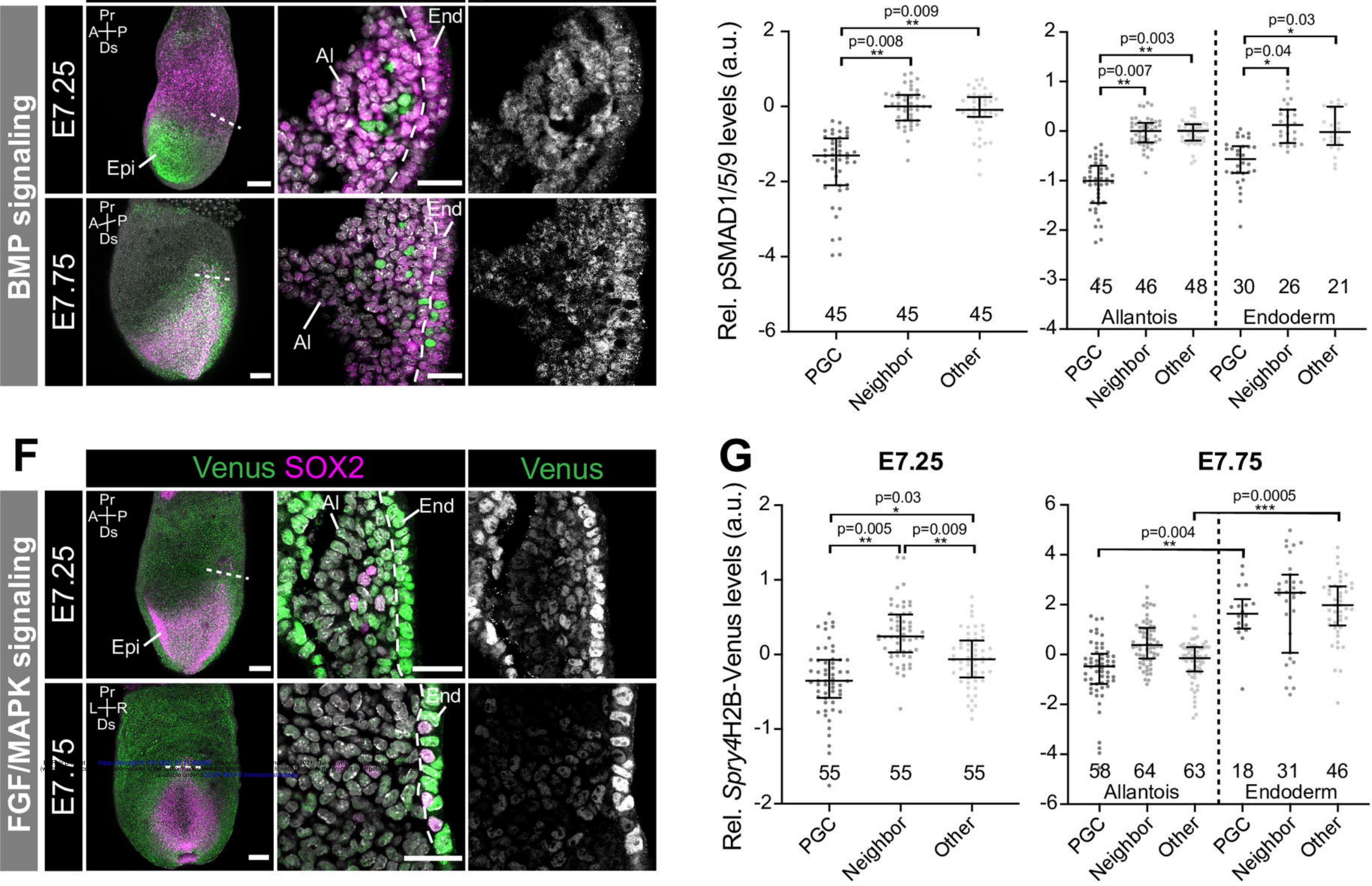

H
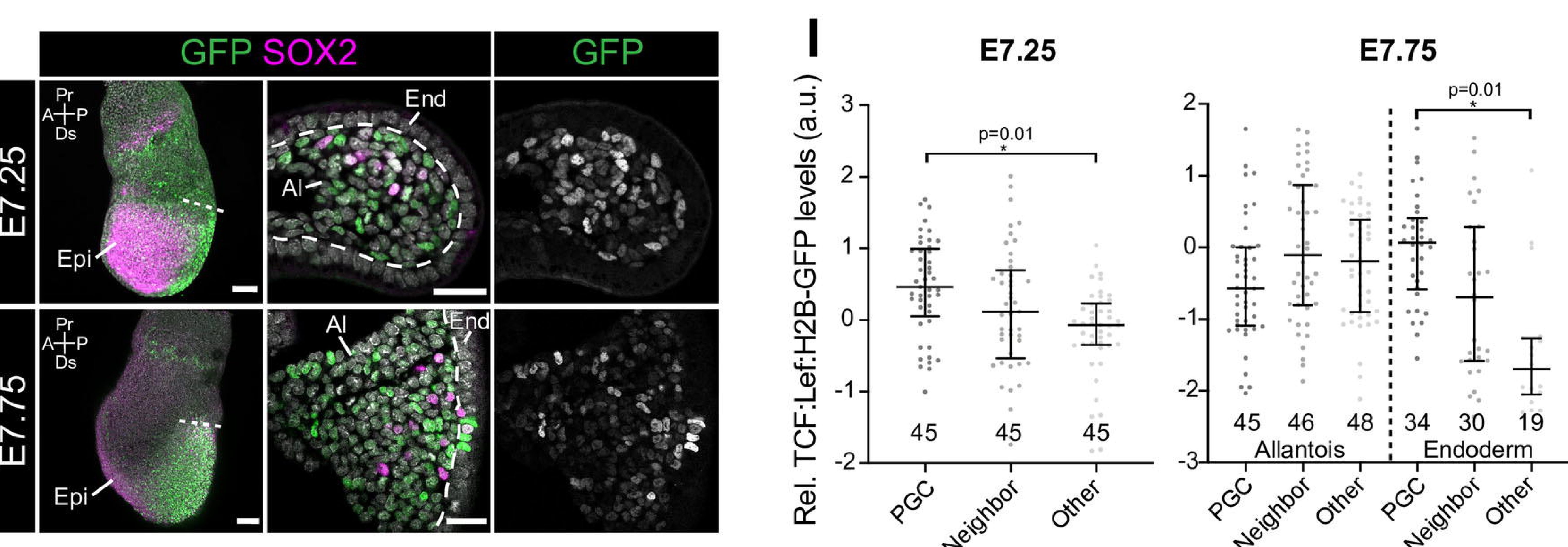
Morgani et al. Figure 3

A

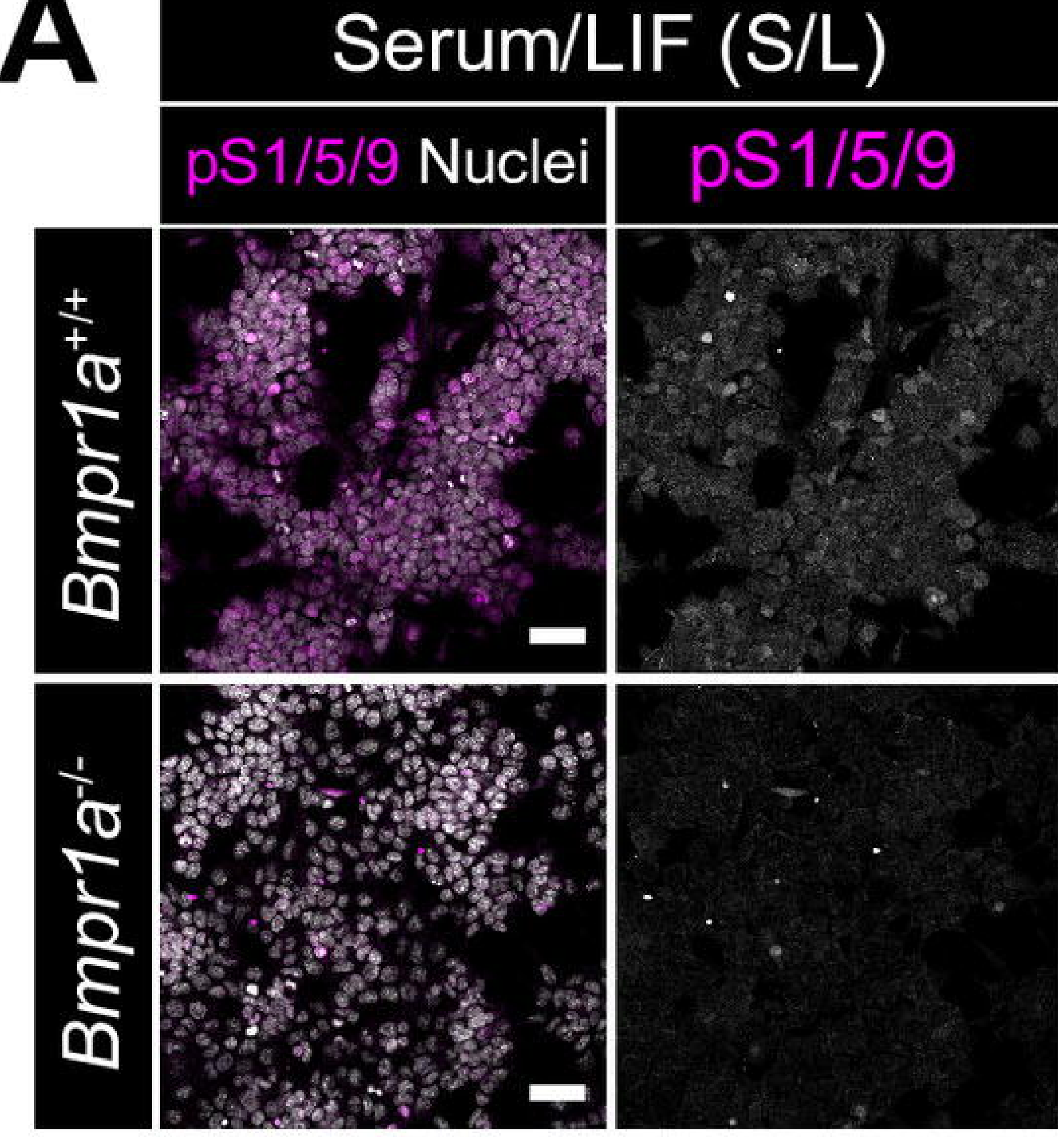

D

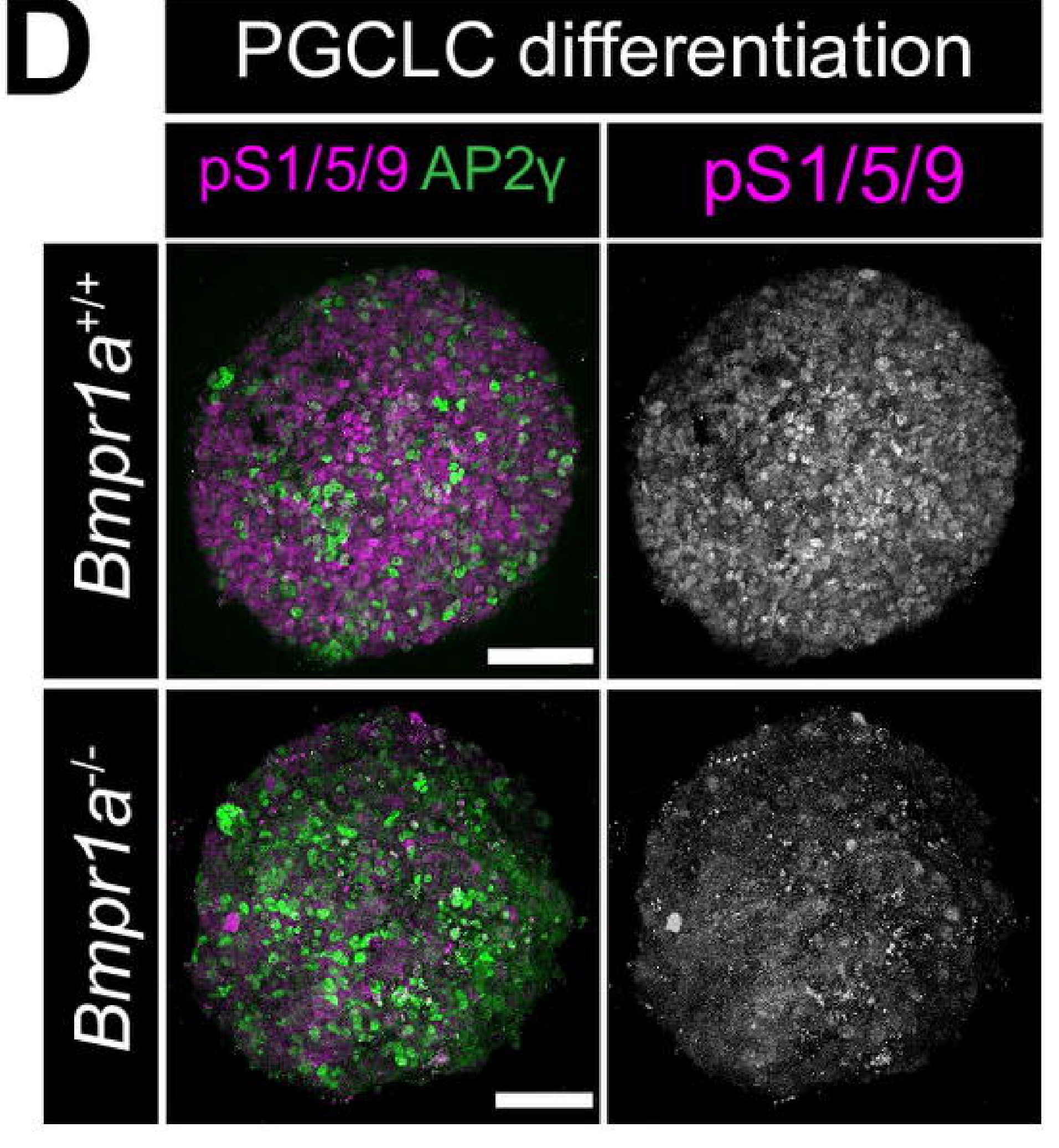

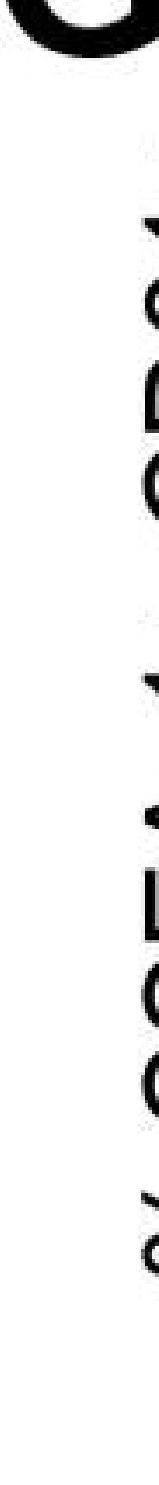

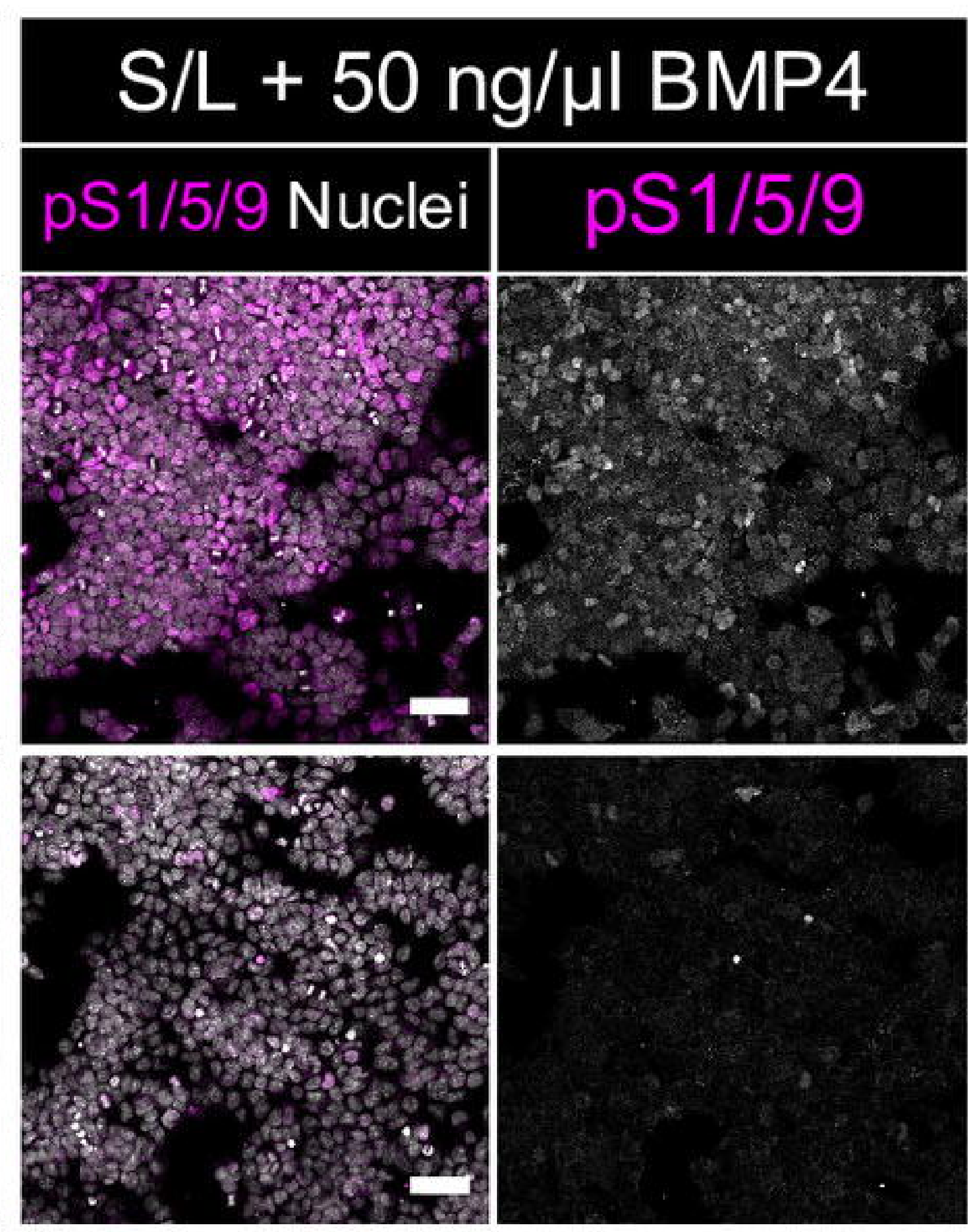

E $\mathrm{Bmpr1a}^{+/+} \mathrm{Bmpr1a}^{-/}$

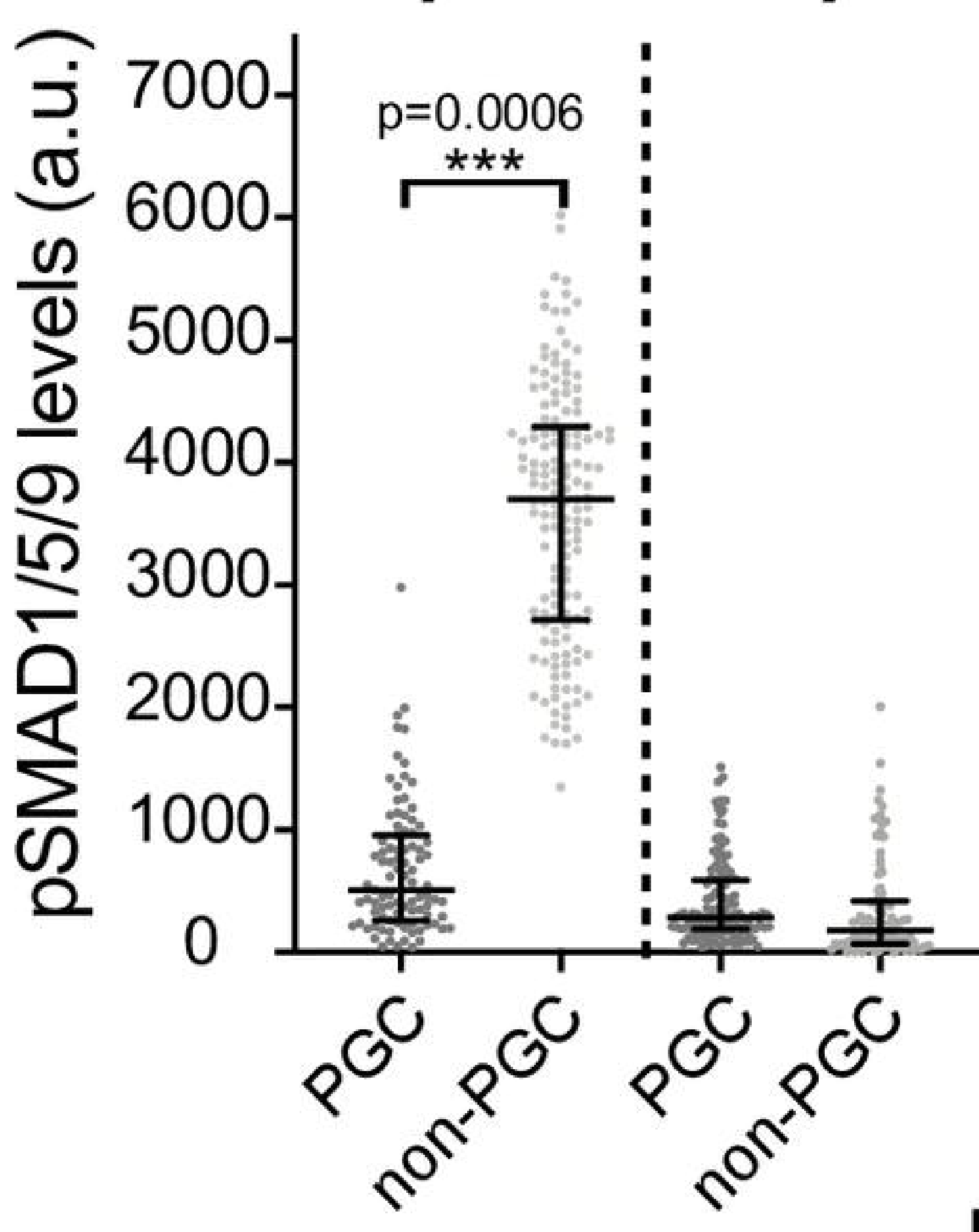

I

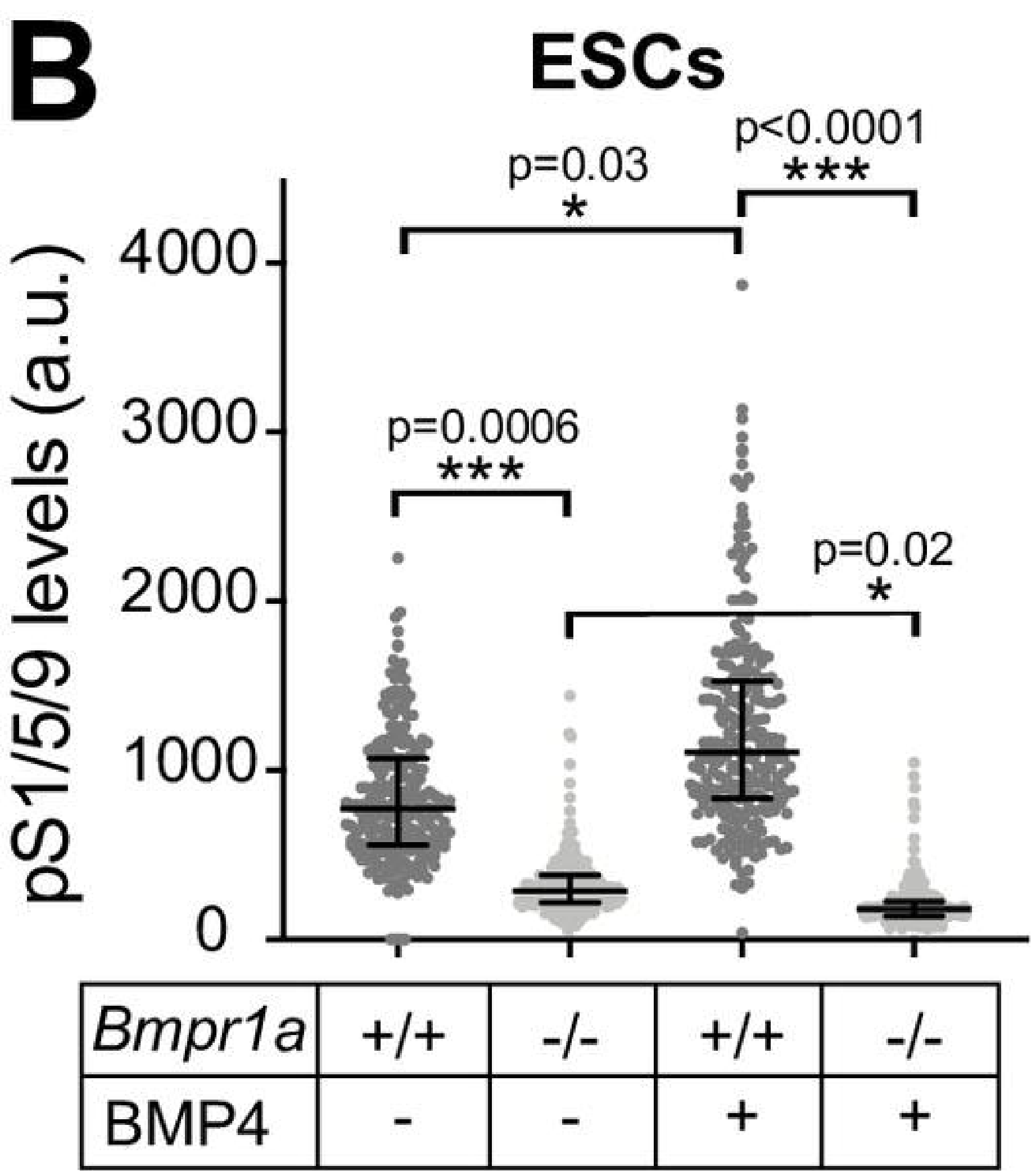

$\mathbf{F}$

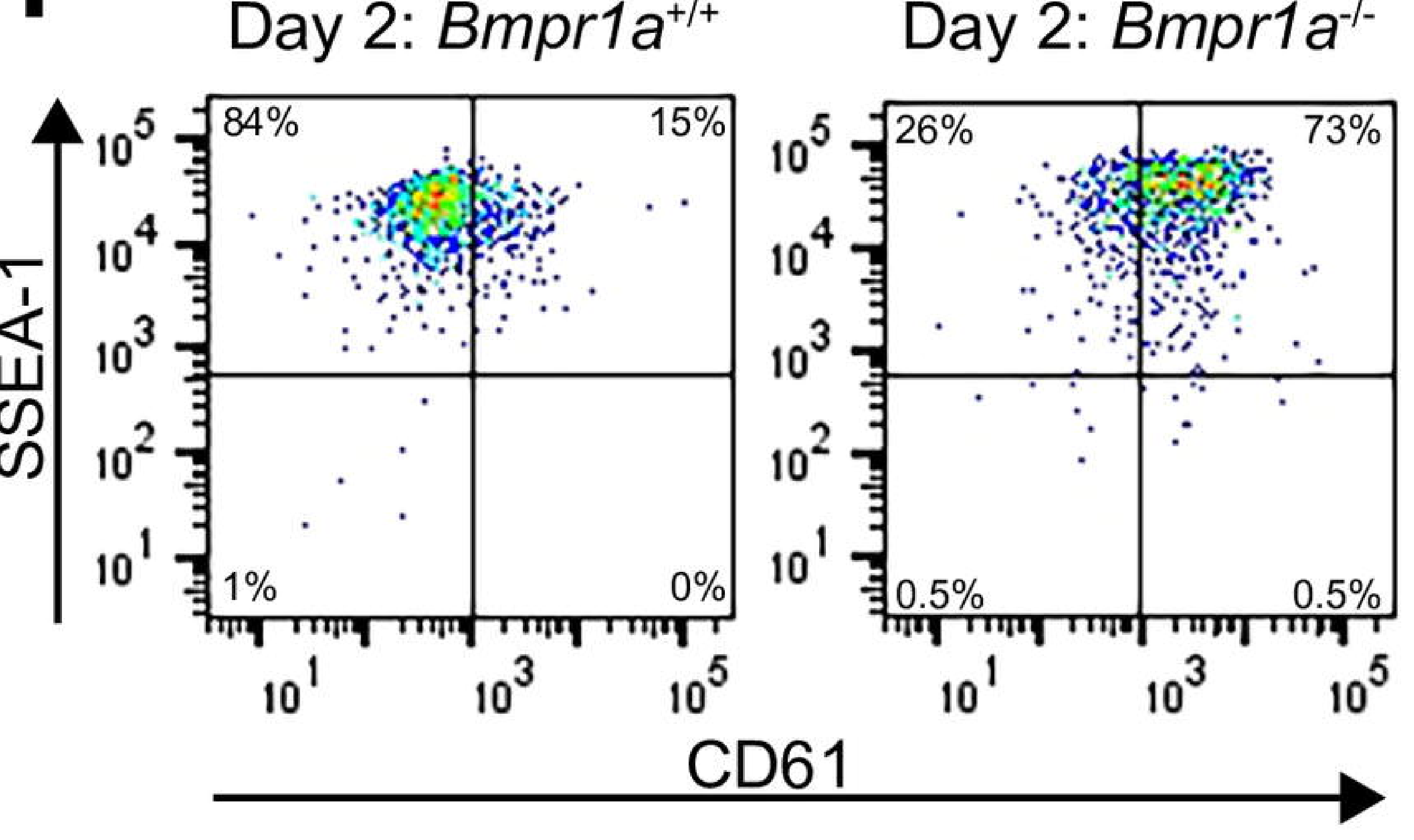

EpiLCs

Wildtype Bmpr1a ${ }^{-1} \quad$ differentiation

Bmpr1a ${ }^{+/+}$ बहै $50: 50$ बहै

- Bmpr1a
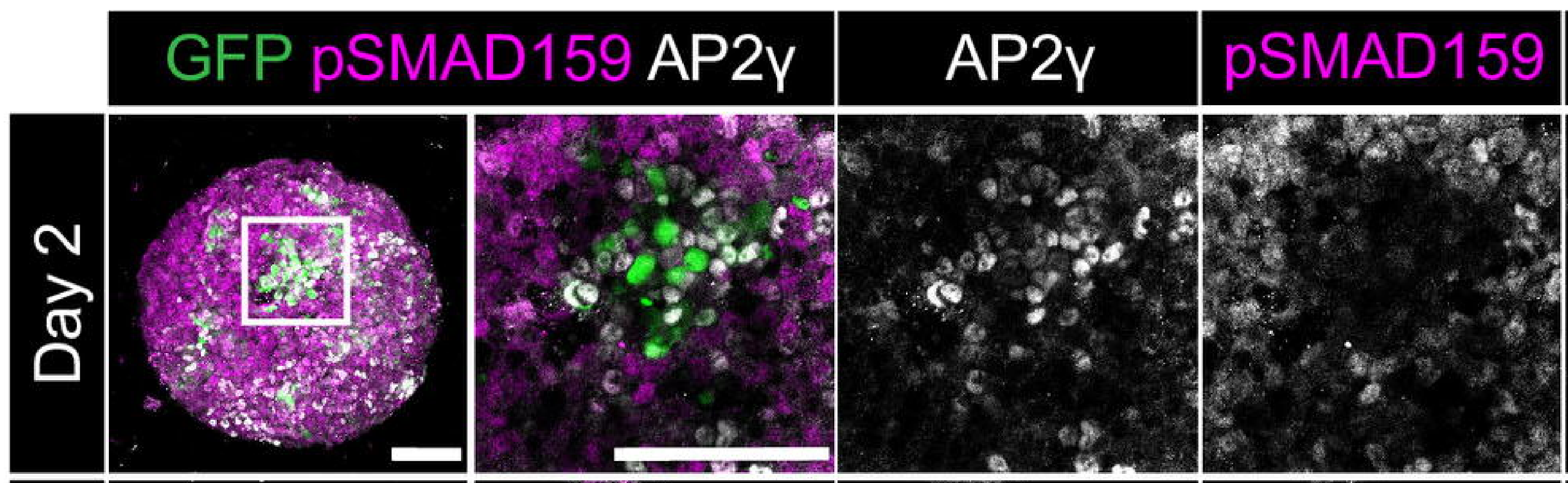

GFP

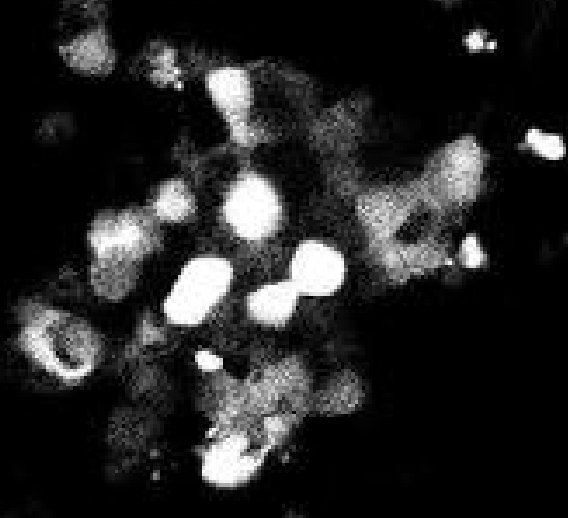

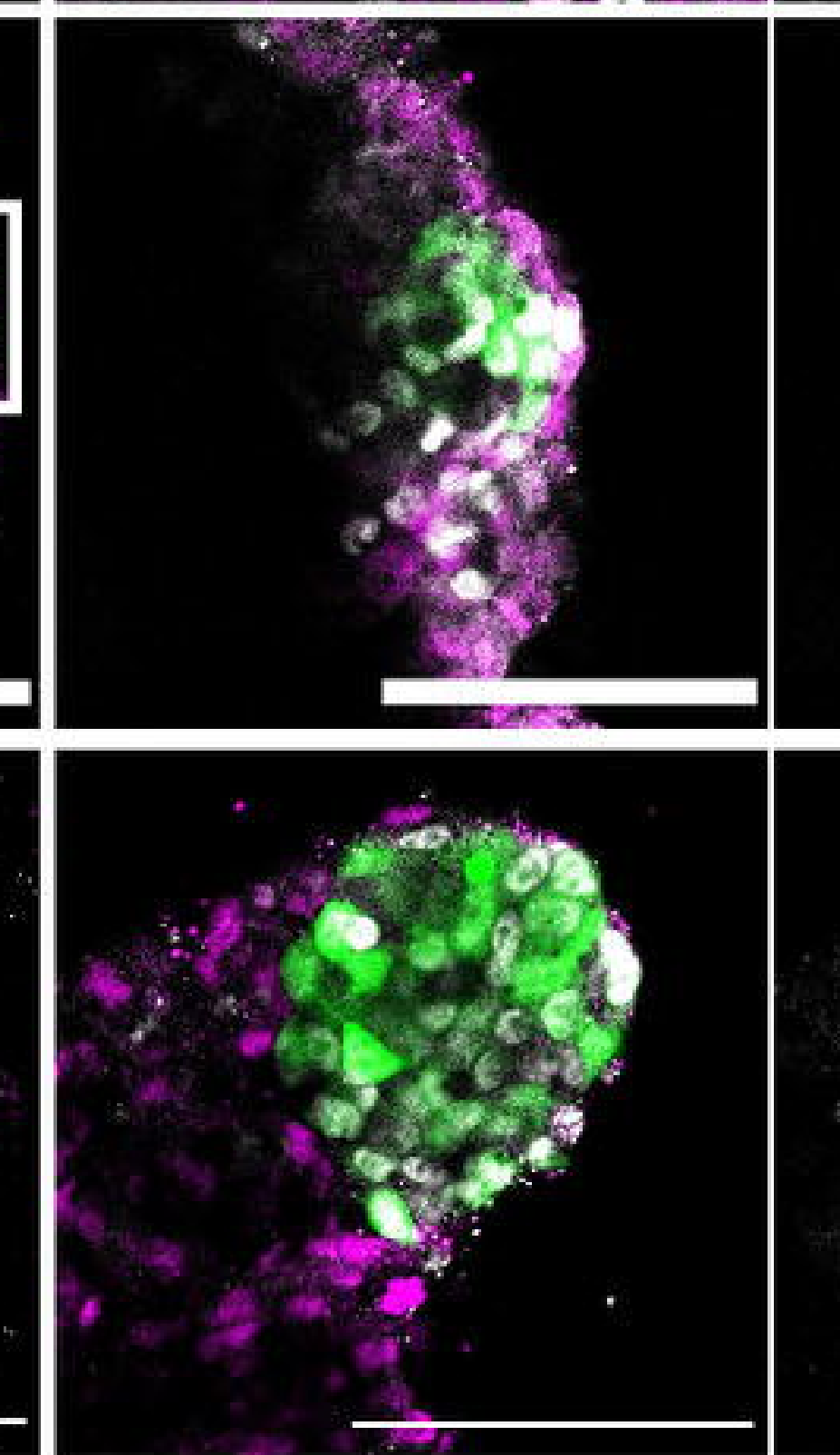

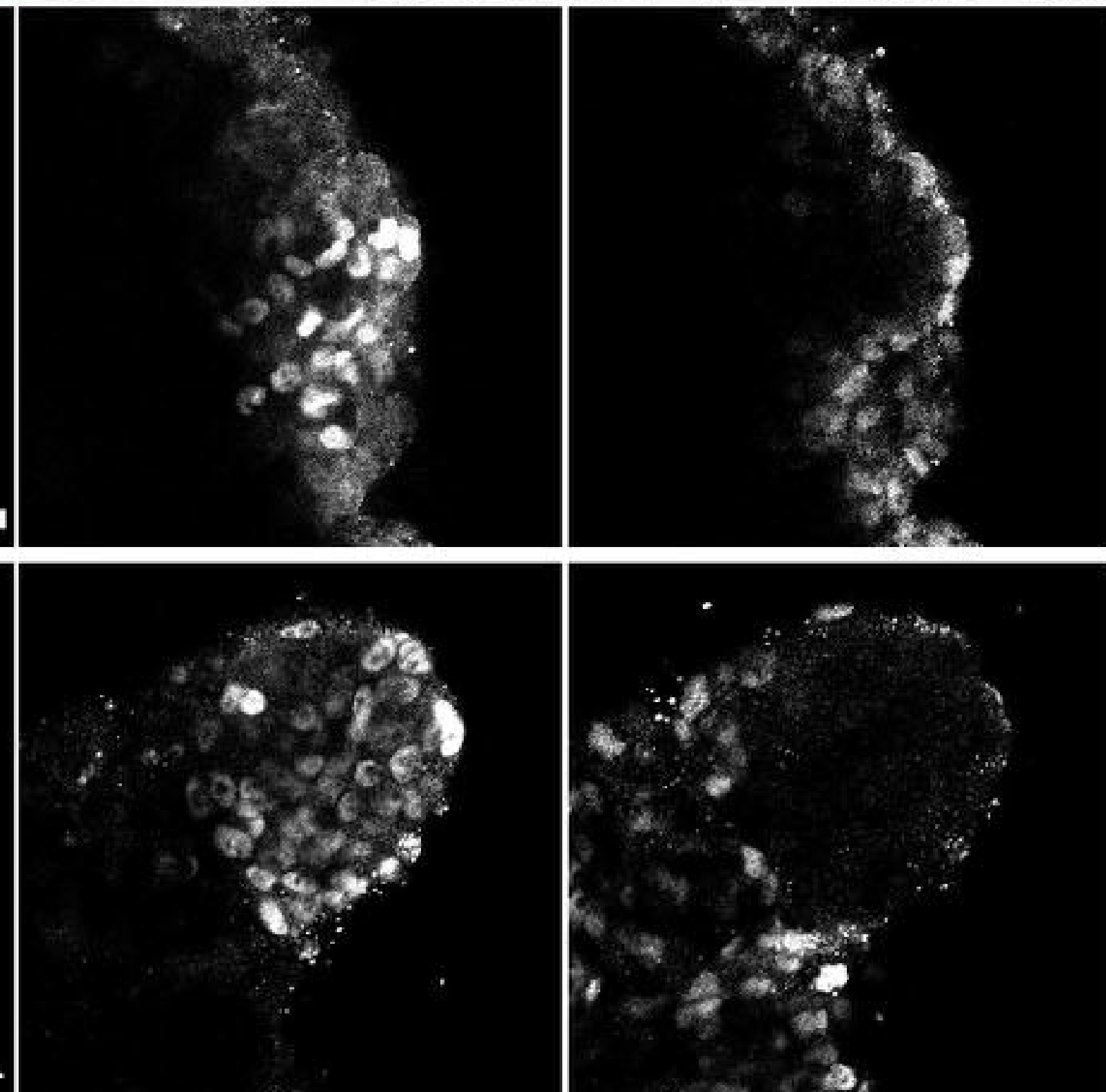

Log AP2y levels (a.u.) 